\title{
Xylo-oligosaccharides from sugarcane show prebiotic potential in a dynamic computer-controlled in vitro model of the adult human large intestine
}

\author{
K. Venema ${ }^{1,2 *}$ J. Verhoeven ${ }^{1}$, S. Verbruggen ${ }^{1}$ and D. Keller ${ }^{3}$ \\ ${ }^{1}$ Centre for Healthy Eating \& Food Innovation (HEFI), Maastricht University - campus Venlo, St. Jansweg 20, 5928 RC \\ Venlo, the Netherlands; ${ }^{2}$ School of Nutrition and Translational Research in Metabolism (NUTRIM), Department of Human \\ Biology, Maastricht University, Universiteitssingel 50, 6229 ER Maastricht, the Netherlands; ${ }^{3}$ Keller Consulting Group, \\ 2417 Beachwood Blvd., Beachwood, OH 44122, USA; k.venema@maastrichtuniversity.nl
}

Received: 17 September 2019 / Accepted: 30 January 2020

(c) 2020 Wageningen Academic Publishers

\begin{abstract}
OPEN ACCESS C(1) @()
RESEARCH ARTICLE

Abstract

The aim of the study was to investigate the prebiotic potential of xylo-oligosaccharides (XOS) from sugarcane in a validated, dynamic, computer-controlled in vitro model of the colon (TIM-2) simulating human adults. In two sets of experiments, each with a different microbiota, 3 different doses of XOS were tested at $1.0 \mathrm{~g} /$ day, $1.5 \mathrm{~g} / \mathrm{day}$ and $3.0 \mathrm{~g} /$ day. The in vitro model was run for $72 \mathrm{~h}$, and at the start and subsequently every $24 \mathrm{~h}$ samples were taken and analysed for short-chain fatty acids (SCFA) and gut microbiota composition. SCFA were analysed using ion chromatography, whereas microbiota composition was analysed using sequencing of the V3-V4 region of the $16 \mathrm{~S}$ rRNA gene. XOS showed a similar SCFA production per gram of substrate as the control medium, including butyrate, which is considered to be important for gut health. In both sets of experiments XOS showed a consistent dose-dependent increase in abundance over time of the genus Bifidobacterium, and within that of the species $B$. adolescentis and an unidentified species (labelled 'sp.1'). The results show the potential prebiotic effect of XOS from sugarcane, by its capacity to generate butyrate and increase the health-beneficial bifidobacteria.
\end{abstract}

Keywords: TIM-2, prebiotic, xylo-oligosaccharides, in vitro model, Bifidobacterium

\section{Introduction}

Prebiotics have recently been redefined as 'a substrate that is selectively utilised by host microorganisms conferring a health benefit' (Blatchford et al., 2013; Gibson et al., 2017). Previously, this was restricted primarily to dietary carbohydrates, gut microbiota and gastrointestinal health. The new definition expands the concept of prebiotics to include non-carbohydrate substances, and applications to other body sites. However, currently most existing and newly investigated potential prebiotics are still largely restricted to carbohydrates. Whereas in the past the term prebiotic was considered to be identical to a bifidogenic effect, currently the health effects are considered to be broader, and may include modulation of short-chain fatty acid (SCFA) production, increases (beneficial) and decreases (pathogenic) in other members of the gut microbiota besides bifidobacteria, and effects related to a number of different diseases and disorders (Blatchford et al., 2013; Gibson et al., 2017). The most commonly used prebiotics are fructo-oligosaccharides (FOS), inulin, lactulose and galacto-oligosaccharides (GOS). However, a number of other plant-derived carbohydrates are being investigated as potential prebiotics. These include arabinoxylo-oligosaccharides (AXOS) from wheat, as well as xylo-oligosaccharides (XOS) from hemp and sugarcane. Since the definition of prebiotics includes a health benefit for the host, health effects of these should be investigated in the host, mostly humans. However, screening new potential prebiotics in human individuals is very expensive, and for this purpose sophisticated, dynamic, computer-controlled in vitro models of the large intestine have been developed (Venema and Van den Abbeele, 2013). Advantages of these dynamic models include: (1) sampling over time at the site where fermentation occurs, and (2) collection of all microbial metabolites that are produced, allowing 
mechanistic insight, which is largely absent from human clinical trials (Venema and Van den Abbeele, 2013). The Netherlands Organisation for Applied Scientific Research (TNO) validated, dynamic, computer-controlled in vitro model of the colon (TIM-2) has several distinct features (Minekus et al., 1999), which makes it a very adequate model to study these mechanisms. The model has been validated with respect to microbial composition and activity (Venema et al., 2000), and has been extensively used in prebiotic research, e.g. with inulin of different degree of polymerisation (Aguirre et al., 2016; Van Nuenen et al., 2003), lactulose (Venema et al., 2003), GOS (Maathuis et al., 2012), and so on.

The aim of the current study was to determine whether XOS derived from sugarcane had a potential prebiotic effect as evaluated using TIM-2. Prebiotic activity was determined as changes in composition of the gut microbiota or changes in its activity (with respect to production of SCFAs). A gut microbiota of healthy adults was used in this study.

\section{Materials and methods}

\section{Test products}

The xylo-oligosaccharides (Prenexus XOS ${ }^{\mathrm{mm}}$ ) from sugarcane were provided by Prenexus Health, Inc. (Gilbert, AZ, USA). The product was composed of $83 \%$ XOS and $0 \%$ free sugars with a degree of polymerisation (DP) range of 3 to 14 (data not shown).

\section{TIM-2 in vitro model of the colon}

TIM-2 simulates the large intestine or colon and consists of 4 interconnected compartments containing flexible membranes, in which peristaltic movements are mimicked by applying pressure on the flexible membrane. Average conditions of healthy human individuals were simulated including: body temperature, $\mathrm{pH}$ in the lumen, composition and rate of secretion fluids, delivery of a predigested substrate from the 'ileum', mixing and transport of intestinal contents by peristalsis, absorption of water and microbial metabolites through the means of a dialysis system, and presence of a complex, high density, metabolically active and anaerobic microbiota of healthy human adults. The dialysis system is a crucial component of the model as it prevents accumulation of microbial metabolites, which would otherwise inhibit or even kill the members of the gut microbiota. The model has been described in detail by Cuevas-Tena et al. (2019). The model was inoculated with a standardised microbiota of healthy adult human volunteers (Venema et al., 2000). For this, faeces were collected from 7 volunteers and pooled in an anaerobic cabinet to allow for a standardised microbiota that could be used throughout the experiments and allowed for comparison between substrates. We have shown before that pooling the microbiota from different individuals leads to a pool with the same metabolic capacity as observed in the individual samples (Aguirre et al., 2014). The pooled microbiota was aliquoted, snap frozen in liquid nitrogen and stored at $-80^{\circ} \mathrm{C}$ until inoculation in the model. Four units were run in parallel. The different doses of the substrates were fed to the microbiota over a period of 3 days after an adaptation period of $16 \mathrm{~h}$ through the feeding syringe. More details are provided in the Supplementary Materials S1, including the composition of the standard medium and dialysis solution.

\section{Experimental set-up}

Two sets of experiments were performed. In the first experiment, 1.5 and $3.0 \mathrm{~g} /$ day of xylo-oligosaccharides were used and compared to the standard medium used in TIM2. A dose-dependency was observed in these experiments (see Results). A second set of experiments was performed, with a different microbiota $(n=7$, of which 5 volunteers overlapped with the first batch) and an even lower dose of $1.0 \mathrm{~g} /$ day was added. The xylo-oligosaccharides replaced the standard carbohydrates used in the control medium, simulated ileal efflux medium (SIEM).

\section{Sampling}

Samples were taken every $24 \mathrm{~h}$ for a period of $72 \mathrm{~h}$ from both the lumen $(\mathrm{n}=4)$ and the dialysate $(\mathrm{n}=2)$ of the system (at 0, 24, 48 and $72 \mathrm{~h}$ ). Samples from both the lumen and dialysate were analysed for SCFA concentrations. The production of metabolites at the moment of addition of the substrates was artificially set to zero, and cumulative production of the different SCFAs was calculated from that moment onwards. Samples from the lumen of the model were analysed for composition of the microbiota by sequencing the V3-V4 region of the $16 \mathrm{~S}$ rRNA gene using Illumina sequencing as described below. The remainder of unused the samples was stored as back-up.

\section{Short chain fatty acid analyses}

Both lumen $(1.5 \mathrm{ml})$ and dialysate $(2 \mathrm{ml})$ samples were centrifuged at $14,000 \times g$ for $10 \mathrm{~min}$, filtered through a $0.45 \mu \mathrm{m}$ PFTE filter, and diluted in the mobile phase (1.5 $\mathrm{mM}$ aqueous sulfuric acid). The analysis was carried out by ion exclusion chromatography (IEC) using an 883 chromatograph (IC, Metrohm, Herisau, Switzerland) equipped with a Transgenomic IC Sep ICE-ION-300 column $(30 \mathrm{~cm} \times 7.8 \mathrm{~mm} \times 7 \mu \mathrm{m}$; Transgenomic, Omaha, NE, USA) and a MetroSep RP2 Guard (Metrohm). $10 \mu \mathrm{l}$ were loaded onto the column with the help of an automatic sampler 730 (Metrohm). A column flow of $0.4 \mathrm{ml} / \mathrm{min}$, with a column temperature of $65^{\circ} \mathrm{C}$ was used. The SCFA were eluted according to their $\mathrm{pKa}$ and were detected using suppressed conductivity detection. 


\section{Next-generation sequencing of V3-V4 region of the $16 \mathrm{~S}$ rRNA gene}

Samples for microbiota composition were analysed by sequencing PCR amplicons of the V3-V4 region of the $16 \mathrm{~S}$ rRNA gene. Briefly, the isolation of genomic DNA from the faecal samples ( $1 \mathrm{ml}$ lumen sample) was performed using standard molecular biology kits from ZYMO Research as provided by the manufacturer (BaseClear, Leiden, the Netherlands). The PCR amplification of the V3 and V4 regions, the barcoding and the library preparation were carried out according to established protocols provided by Illumina (Illumina, Eindhoven, the Netherlands). The sequencing was carried out using the Illumina MiSeq system (San Diego, CA, USA) and later the sequences were converted into FASTQ files using the BCL2FASTQ pipeline version 1.8.3. The quality cut was applied based on the Phred quality score. QIIME software package (1.9.0) was used for microbial analyses (Caporaso et al., 2010). The sequences were classified using Greengenes (version 13.8) as a reference $16 \mathrm{~S}$ rRNA gene database.

\section{Statistical analyses}

Correlations between Operational Taxonomic Units (OTUs) and test-products were investigated using the KruskalWallis test, by using the software package $\mathrm{R}$ (3.5.3) (R Core Team, 2013) in RStudio. Multiple comparison was corrected using the false discovery rate (FDR), and $q$-values (adjusted $P$-values) were considered significantly different at a strict cut-off of $P<0.05$. PERMANOVA was used for significance testing on distance matrices (Anderson, 2001).

\section{Results and discussion}

\section{Production of microbial metabolites}

The cumulative average production of SCFA, expressed as $\mathrm{mM} / \mathrm{g}$ of carbohydrate fed, for the first set of experiments is indicated in Figure 1A. Compared to the standard medium (SIEM), the production of SCFA was very similar, with slightly higher cumulative amounts for the low dose of XOS (1.5 g/day). On both the 1.5 and 3.0 g per day doses, more lactate was produced (Supplementary Table S1) when XOS was fed, compared to the standard medium. In the second set of experiments, where a different microbiota was used, similar results were obtained, although in this case the standard medium showed the highest cumulative amount. The $1.0 \mathrm{~g}$ per day dose showed slightly higher cumulative SCFA production than the $1.5 \mathrm{~g}$ per day dose. It is not unusual to observe a higher cumulative SCFA of a production per gram of substrate added in a dose-response. Although not completely understood, this may be because of cross feeding between the members of the microbiota, which is different at different doses of substrate. The inoculum in the $2^{\text {nd }}$ experiment overall showed a slightly lower butyrate production than the $1^{\text {st }}$ experiment. In the $2^{\text {nd }}$ experiment the cumulative butyrate production for the 1.0 and $3.0 \mathrm{~g} /$ day doses were slightly lower than for the control medium and the $1.5 \mathrm{~g} /$ day dose. But other than that, the production of SCFA, expressed as per gram of substrate added, was very similar. Lactate production was slightly higher upon XOS feeding as compared to feeding the control medium (Supplementary Table S1). This is in agreement with the increase in abundance of bifidobacteria (see below). Bifidobacteria produce lactate in addition to acetate. Lactate is considered an intermediate metabolite, and only accumulates if fermentation is fast. If fermentation is slow, it is converted into acetate, propionate or butyrate (Morrison et al., 2006). Butyrate is considered to be one of the most important metabolites produced by the gut microbiota since it is a substrate for colonocytes (Hamer et al., 2008). And, although bifidobacteria primarily produce acetate and lactate, other members of the gut microbiota can use either acetate or lactate through cross-feeding and convert these into butyrate (Kovatcheva-Datchary et al., 2009; Morrison et al., 2006). The fact that XOS feeding leads to similar amounts of butyrate as produced on the control medium, indicates that XOS may contribute to gastrointestinal health as a good substrate for production of butyrate.

With gut microbiology it is very difficult to use a placebo for a fermentable substrate. This is because the gut microbes always want to ferment a substrate to generate energy, to survive and multiply. Some researchers have used cellulose (which for human microbiota is considered mostly unfermentable (Slavin et al., 1981)). In this case, the microbiota will perhaps not ferment the cellulose, but they will switch to protein fermentation, as they need energy to survive. Therefore, to use cellulose leading to mostly protein fermentation is not a good 'placebo' for a prebiotic either. Thus, the best control would be a mixed undigestible fibre containing SIEM medium, that reflects the normal dietary fibre intake (Aguirre et al., 2016; Van Nuenen et $a l ., 2003)$. There appears to be no appreciable difference over SIEM with respect to SCFA production. This means that the gut ecology under the conditions imposed in the in vitro model is not dramatically disturbed. The community in the gut should be 'in balance', and therefore a drastic change induced by dietary changes will not be maintained, as this will shift the 'balance' (or homeostasis). Despite some changes in microbiota composition (see next paragraph), this does not lead to a (drastic) change in the ecological balance in the in vitro model, and moreover does not seem to affect the overall metabolic activity of the microbial consortium under the current conditions. 


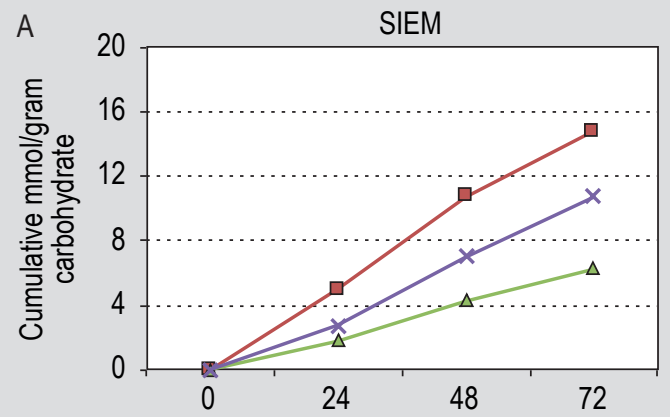

$$
\begin{aligned}
& - \text { acetate } \\
& \Delta \text { propionate } \\
& * \text { butyrate }
\end{aligned}
$$
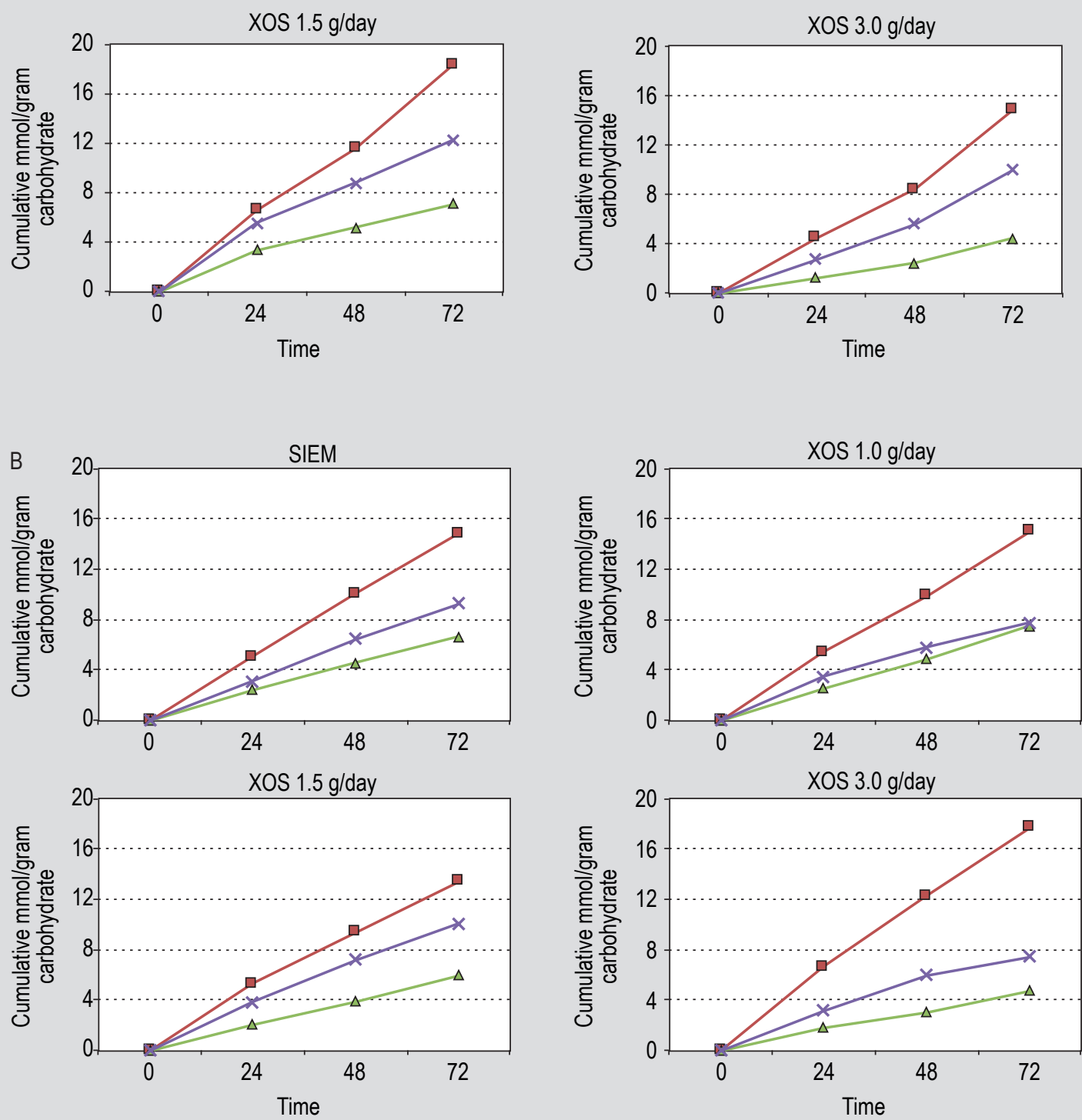

Figure 1. Cumulative production of short chain fatty acids (SCFA), expressed as SCFA produced per gram of carbohydrate provided per day. (A) First set of experiments; (B) second set of experiments. SIEM = simulated ileal efflux medium; XOS = xylooligosaccharides. 
Table 1. $P$-values, $q$-values and operational taxonomic units (OTUs) that correlate with the different substrates fed.

\begin{tabular}{|c|c|c|c|}
\hline & $P$-value & q-value ${ }^{1}$ & OTU \\
\hline \multirow[t]{8}{*}{$1^{\text {st }}$ set of experiments } & 0.000476 & 0.011 & Bifidobacterium \\
\hline & 0.000564 & 0.011 & Paraprevotella \\
\hline & 0.000448 & 0.011 & Faecalibacterium \\
\hline & 0.000315 & 0.011 & Ruminococcus \\
\hline & 0.000838 & 0.012 & Citrobacter \\
\hline & 0.001795 & 0.020 & Roseburia \\
\hline & 0.002965 & 0.029 & Lachnospira \\
\hline & 0.003589 & 0.031 & Prevotella \\
\hline \multirow[t]{20}{*}{$2^{\text {nd }}$ set of experiments } & $5.7 \times 10^{-06}$ & 0.00029 & Lactococcus \\
\hline & $3.5 \times 10^{-06}$ & 0.00029 & Lachnospira \\
\hline & 0.000105 & 0.0027 & Bifidobacterium \\
\hline & 0.00026 & 0.0054 & Blautia \\
\hline & 0.000475 & 0.0077 & Ruminococcaceae;g__ \\
\hline & 0.001086 & 0.011 & Clostridium \\
\hline & 0.001029 & 0.011 & Faecalibacterium \\
\hline & 0.000989 & 0.011 & Enterobacteriaceae;other \\
\hline & 0.001543 & 0.013 & Collinsella \\
\hline & 0.001461 & 0.013 & Phascolarctobacterium \\
\hline & 0.001882 & 0.014 & Lachnospiraceae;other \\
\hline & 0.001856 & 0.014 & Ruminococcus \\
\hline & 0.002919 & 0.020 & Paraprevotellaceae;g__ \\
\hline & 0.003031 & 0.020 & Dorea \\
\hline & 0.004595 & 0.027 & Prevotellaceae;g__ \\
\hline & 0.00464 & 0.027 & Enterobacteriaceae;g_ \\
\hline & 0.004996 & 0.027 & Cyanobacteria;c_4COd-2;0_YS2;f_;g__ \\
\hline & 0.005811 & 0.030 & Bacteroides \\
\hline & 0.006295 & 0.031 & Coriobacteriaceae;g__ \\
\hline & 0.008283 & 0.039 & Roseburia \\
\hline
\end{tabular}

\section{Changes in microbiota composition}

The composition of the gut microbiota in the samples was evaluated by sequencing the V3-V4 region of the $16 \mathrm{~S}$ rRNA gene. The $\beta$-diversity of the samples from the first set of experiments is plotted in the principal coordinate analysis $(\mathrm{PCoA})$ indicated in Figure 2A. The red-coloured samples on the far left in each panel are the time 0 samples, after the adaptation phase in which SIEM was fed. At that time the interventions with XOS (1.5 and $3.0 \mathrm{~g} /$ day) were initiated and compared to the control in which SIEM was added. Figure 2A shows that the samples in which the control medium was fed (in red) cluster to the top of the graph, whereas the samples that received $1.5 \mathrm{~g} /$ day XOS (orange) and $3.0 \mathrm{~g} /$ day XOS (green) cluster more to the bottom of the graph. This separation in the weighted Unifrac between SIEM- and XOS-fed microbiotas was significant ( $q$-value $=0.017$ after PERMANOVA analysis). Similarly, in the $2^{\text {nd }}$ set of experiments (Figure 2B) the 3 different doses of XOS cluster away from the control medium, indicating changes in the gut microbiota composition; this was also significant ( $q$-value $=0.005)$. It is of course obvious that a single carbohydrate substrate (XOS) has a different effect compared to a multi-substrate medium (SIEM), but the intention of the current study was to mechanistically investigate the effect of XOS, and the advantage of the in vitro approach is that this can be tested in the absence of any carbohydrates in the 'background diet.'

\section{Correlations between test products and microbiota composition}

For the first set of experiments, the Kruskal-Wallis correlation test between the presence of the different OTUs at the genus level and the feeding of the different test products showed that there were some correlations (Table 1, top). The genus Bifidobacterium, with a $q$-value $=0.01$, showed a dose-dependent increase upon XOS 
A

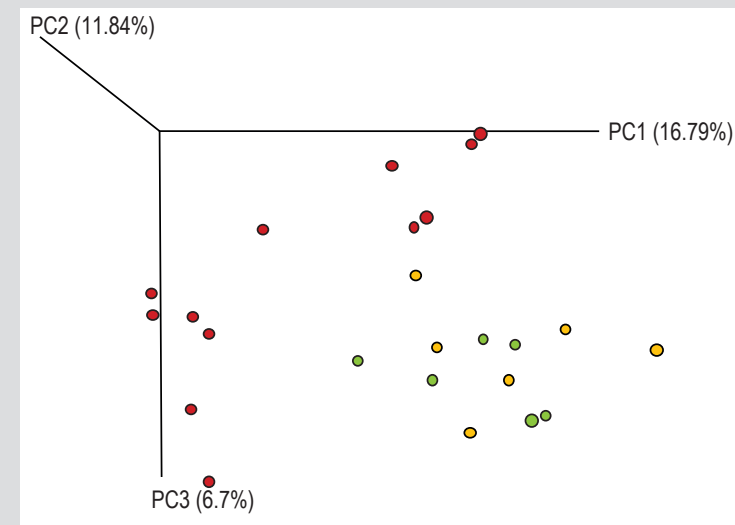

$\square$ SIEM
$B$

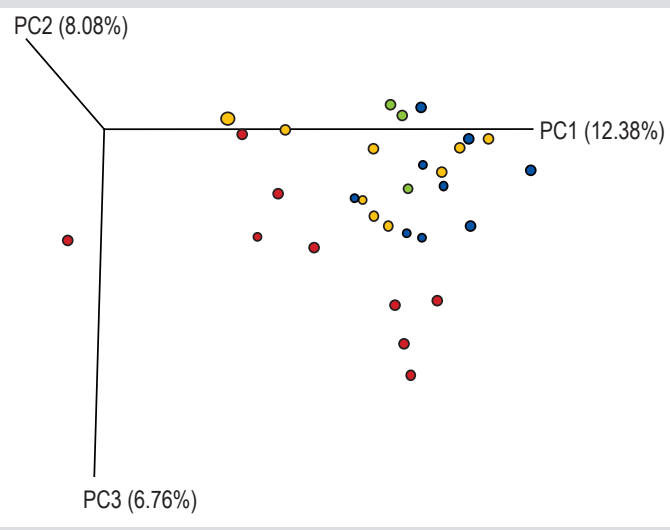

口XOS, 1.5 g/day

$\square \mathrm{XOS}, 3.0$ g/day

Figure 2. Principal component analysis (weighted UniFrac) of the samples, rotated to show the separation between treatments. (A) First set of experiments; (B) second set of experiments. SIEM = simulated ileal efflux medium; XOS = xylo-oligosaccharides.

feeding (Figure 3A). Also, in the second set of experiments Kruskal-Wallis correlation test showed a low $q$-value for Bifidobacterium $(q<0.003$; Table 1, bottom), and a similar dose-dependent effect of XOS was observed (Figure 3B), despite the use of a different microbiota inoculum. As indicated at the top of Table 1 , in the first set of experiments several other microorganisms were significantly different in their abundance in the different variables tested. However, none of these were stimulated. The boxplots for these microorganisms are given in Supplementary Figure S1. Several of the microorganisms that were modulated in the first set of experiments were also shown to be modulated in the second set. These included Ruminococcus, Lachnospira and Faecalibacterium. In the $2^{\text {nd }}$ set of experiments these microorganisms were modulated in a similar manner as in the first set of experiments (see Supplemental Online Material). However, the $2^{\text {nd }}$ set of experiments showed some other microorganisms that were also increased in abundance, such as Lactococcus and Blautia (Figure 3C and D). These 2 OTUs were not modulated in the first set of experiments (data not shown).

The boxplots in Figure 3 show the summed data for all 3 time points at which samples were taken: 24,48 and $72 \mathrm{~h}$ after start of the interventions. Figure $4 \mathrm{~A}$ shows the development of the abundance over time for the 3 variables tested in the first set of experiments. The figure shows that there was a steady increase in abundance for both doses used. Figure 4B shows similar data for the $2^{\text {nd }}$ set of experiments.
The sequencing data also has information on (some) bifidobacterial species. Five individual OTUs can be recognised in the sequence data of the first set of experiments, of which 3 can be classified to the species level: Bifidobacterium adolescentis, Bifidobacterium longum and Bifidobacterium pseudolongum. The other 2 are labelled as 'other' and 'sp.1'. For these 5 species a Student's T-test was performed to look at differences between variables tested (Table 2). B. adolescentis shows a dose-dependent increase when XOS was used (Figure 3E). B. pseudolongum is below the level of detection in SIEM and the $1.5 \mathrm{~g} /$ day dose XOS but appears in a few samples of the high dose XOS (3.0 g/day). For B. longum and the species labelled 'Other' there are no significant differences. However, also the 'sp.1' species shows a significant difference between SIEM and the $3.0 \mathrm{~g} /$ day dose XOS, and a trend between SIEM and the $1.5 \mathrm{~g} /$ day dose XOS (Figure 3F). In the $2^{\text {nd }}$ set of experiments B. pseudolongum was absent, but the same other four species were observed in this inoculum as well. B. adolescentis was again significantly increased compared to the control medium (Table 2).

As for the boxplots for the genus Bifidobacterium, the data for the species shows the sum of the 3 different time points. The development over time of the abundance of B. adolescentis and the Bifidobacterium sp. 1 for the 4 variables tested in the 2 sets of experiments are displayed in Figures 4. Similar to the observation made for the genus Bifidobacterium, B. adolescentis and the other species show a dose-dependent increase, as well as an increase over time, in both sets of experiments. 
A

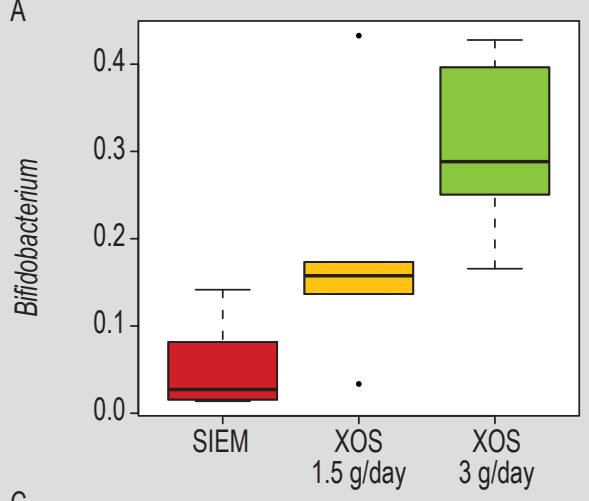

$\mathrm{C}$

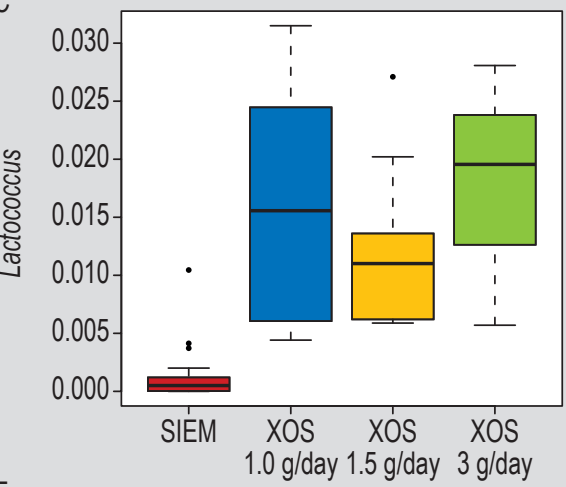

E
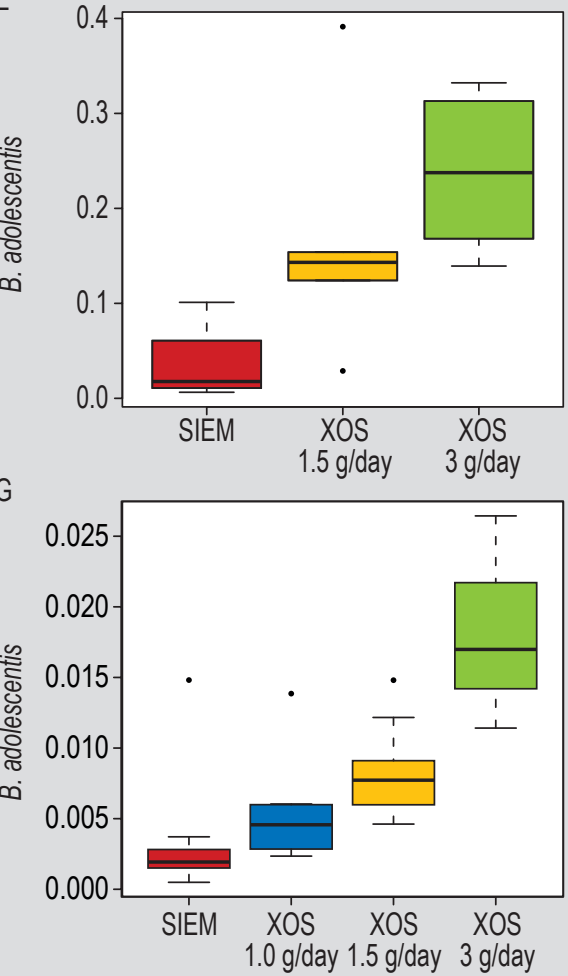

B

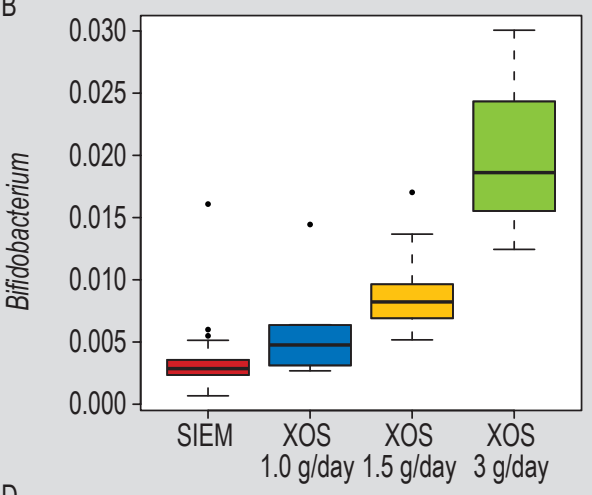

D

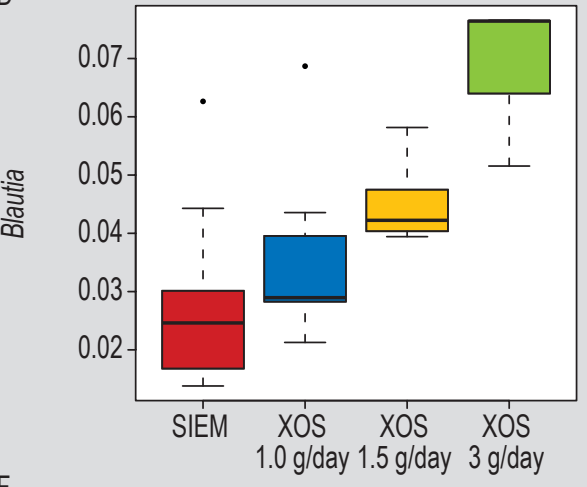

F

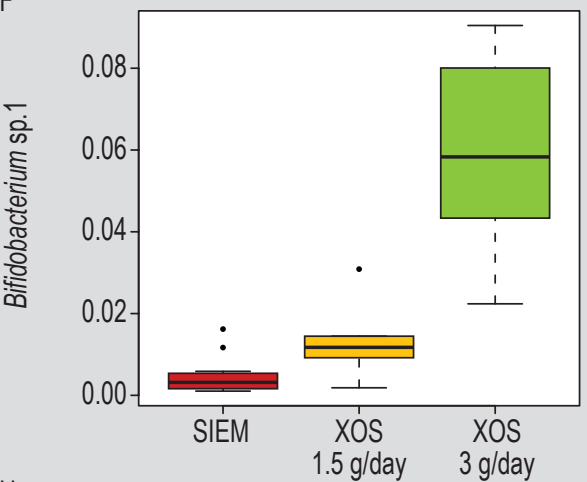

$\mathrm{H}$

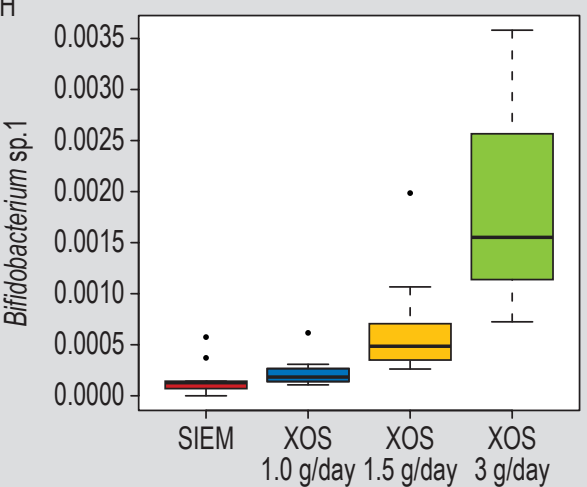

Figure 3. Boxplot of the average abundance of several operational taxonomic units in the samples and genus (A-D) or species (E-H) level. A, E and F: first set of experiments; B, C, D, G and H: $2^{\text {nd }}$ set of experiments. Colour-coding is the same as for the PCoA plots in Figure 2. Data are summed for the time-points T24, T48 and T72. The abundance of each time-point is plotted in Figure 4. $P$-values for comparison between SIEM and the XOS treatments are: SIEM vs XOS-1.5 g/day; $P=0.058$; SIEM vs XOS-3.0 $\mathrm{g} / \mathrm{day} ; P=0.00096$ for the $1^{\text {st }}$ set of experiments (A); SIEM vs XOS-1.0 g/day; $P=0.188 ;$ SIEM vs XOS-1.5 g/day; $P=0.0086$; SIEM vs XOS-3.0 g/day; $P=0.038$ for the $2^{\text {nd }}$ set of experiments $(B)$. SIEM = simulated ileal efflux medium; $X O S=x y l 0-o l i g o s a c c h a r i d e s$. 
Table 2. P-values of Student's T-test between the different treatments for the bifidobacterial species. ${ }^{1}$

\begin{tabular}{|c|c|c|c|c|c|c|}
\hline & \multirow[t]{2}{*}{ Treatments } & \multicolumn{5}{|l|}{$P$-value ${ }^{2}$} \\
\hline & & $\begin{array}{l}\text { Bifidobacterium } \\
\text { adolescentis }\end{array}$ & $\begin{array}{l}\text { Bifidobacterium } \\
\text { longum }\end{array}$ & $\begin{array}{l}\text { Bifidobacterium } \\
\text { pseudolongum }\end{array}$ & 'Other' & $\begin{array}{l}\text { Bifidobacterium } \\
\text { 'sp.1' }\end{array}$ \\
\hline \multirow[t]{2}{*}{$1^{\text {st }}$ set of experiments } & SIEM vs XOS-1.5 g/day & 0.048 & 0.26 & n.d. & 0.18 & 0.08 \\
\hline & SIEM vs XOS-3.0 g/day & $9.9 \times 10^{-4}$ & 0.58 & 0.36 & 0.47 & $2.8 \times 10^{-3}$ \\
\hline \multirow[t]{6}{*}{$2^{\text {nd }}$ set of experiments } & SIEM vs XOS-1.0 g/day & 0.15 & 0.038 & n.d. & 0.033 & 0.20 \\
\hline & SIEM vs XOS-1.5 g/day & 0.008 & 0.39 & n.d. & 0.49 & 0.009 \\
\hline & SIEM vs XOS-3.0 g/day & 0.031 & 0.24 & n.d. & 0.08 & 0.09 \\
\hline & XOS-1.0 g/day vs XOS-1.5 g/day & 0.043 & 0.029 & n.d. & 0.003 & 0.018 \\
\hline & XOS-1.0 g/day vs XOS-3.0 g/day & 0.045 & 0.11 & n.d. & 0.48 & 0.09 \\
\hline & XOS-1.5 g/day vs XOS-3.0 g/day & 0.07 & 0.31 & n.d. & 0.042 & 0.13 \\
\hline
\end{tabular}

It should be noted that the relative abundance of bifidobacteria (at genus and species level) is different between the two sets of experiments. There is an approximately 10 -fold higher relative abundance in the $1^{\text {st }}$ set. This is caused by the higher abundance of bifidobacteria in the pooled inoculum of the $1^{\text {st }}$ set, as can be observed by comparing e.g. Figure $4 \mathrm{~A}$ and $4 \mathrm{~B}$ at time point 0 (relative abundance 0.05 vs 0.005 , respectively).

Bifidobacteria are considered to be health beneficial (Bottacini et al., 2017; Hidalgo-Cantabrana et al., 2017). Strains of this genus are frequently used as probiotics. Ingestion of probiotics, including bifidobacterial strains, has been shown to be beneficial for numerous diseases and disorders. However, probiotics rarely colonise the gut and are washed out after a couple of days. A different strategy is to increase the endogenous populations of bifidobacteria in the gut. These are naturally present in the large intestine, have been selected by the host and its dietary pattern, and are therefore a natural target to increase. As alluded to before, in the past a prebiotic effect was considered to be equal to an increase in bifidobacteria. Classic prebiotics, such as FOS and inulin, and GOS have been shown to increase bifidobacteria (Maathuis et al., 2012; Van Nuenen et al., 2003). Therefore, the observed increases in two bifidobacterial species in the current experiments show the prebiotic potential of XOS from sugarcane. Because the definition of prebiotics includes a health benefit for the host, these effects should be replicated in a human clinical trial, and should be associated with health benefits, before
XOS from sugarcane can really be considered a prebiotic. This will also indicate whether the changes will be observed under more realistic conditions, e.g. in the presence of other carbohydrates from the diet.

\section{Supplementary material}

Supplementary material can be found online at https://doi. org/10.3920/BM2019.0159.

Materials S1. Dialysate and simulated ileal efflux medium.

Figure S1. Boxplot of the average abundance of several operational taxonomic units in the samples.

Table S1. Cumulative lactate production at time-point 72.

Table S2. $P$-values and $q$-values for the different bifidobacterial species from the Kruskal-Wallis test with the interventions.

\section{Acknowledgements}

The study was carried out by the Centre for Healthy Eating \& Food Innovation (HEFI) of Maastricht University campus Venlo. This research was funded by Prenexus Health, Inc., and has been made possible with the support of the Dutch Province of Limburg. 

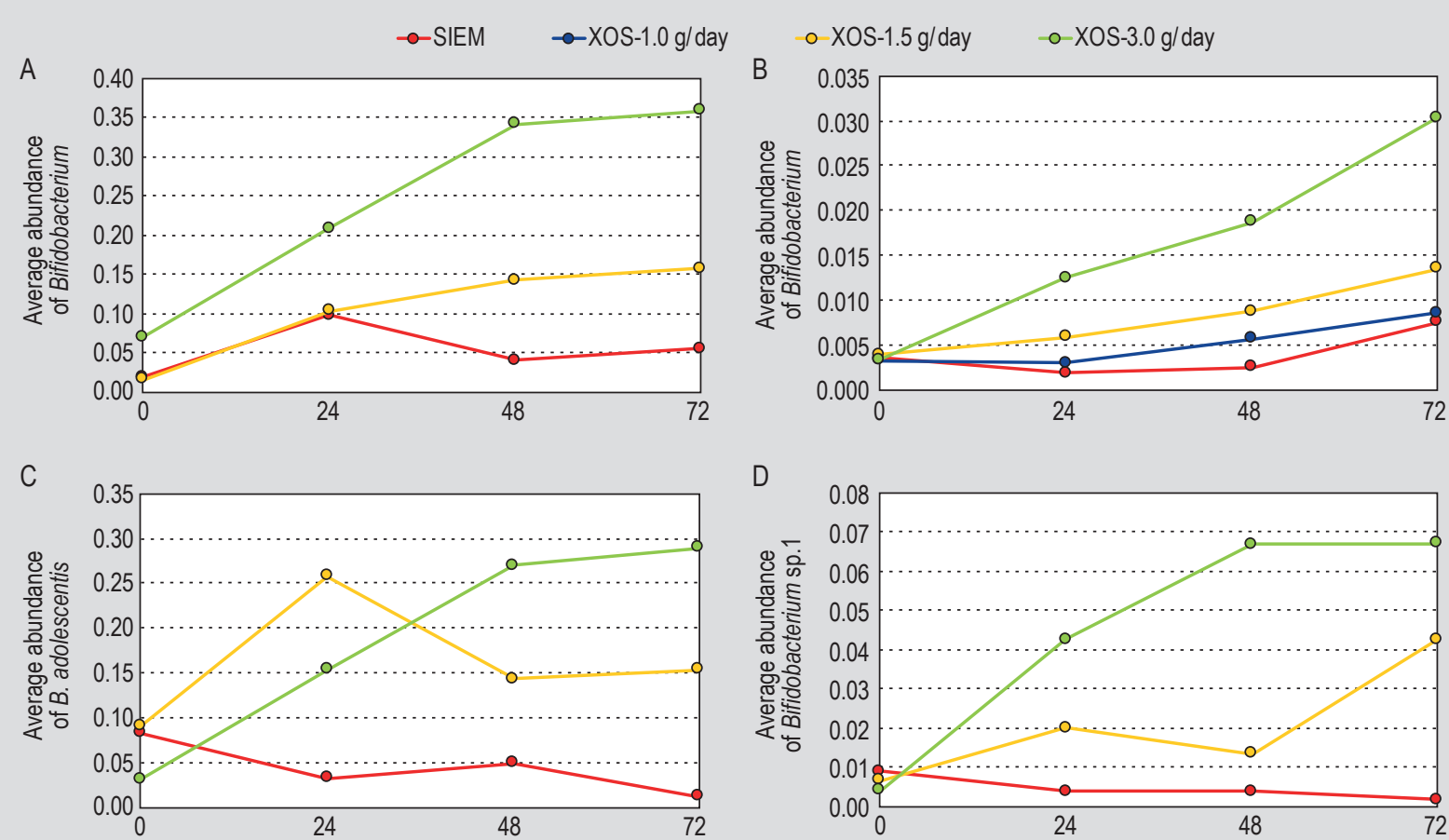

D

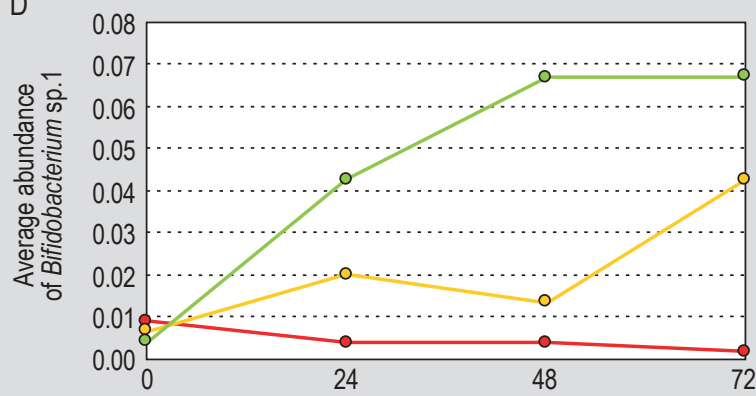

E

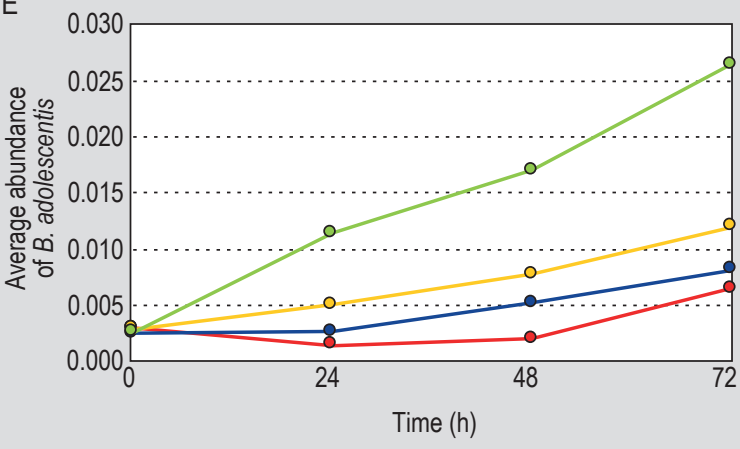

$\mathrm{F}$

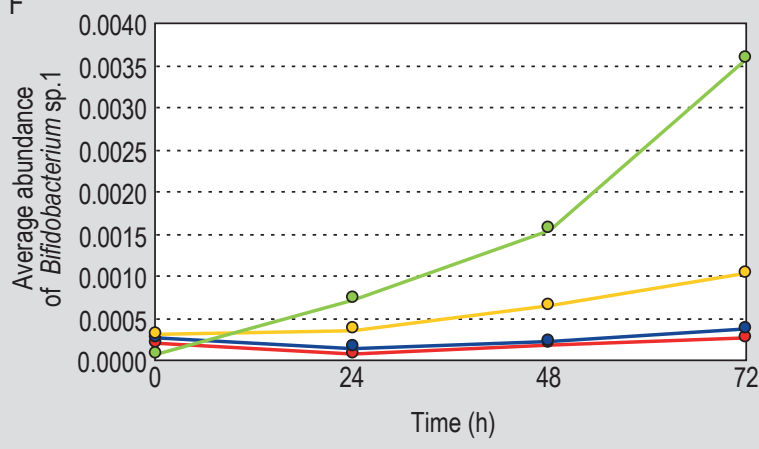

Figure 4. Development of the average abundance for the duplicate runs of the genus Bifidobacterium, and the species Bifidobacterium adolescentis and Bifidobacterium sp.1 over time for the different substrates. A, C and D: first set of experiments; $B, E$ and F: $2^{\text {nd }}$ set of experiments. Colour-coding is the same as for the PCoA plots in Figure 2. SIEM = simulated ileal efflux medium; XOS = xylo-oligosaccharides.

\section{References}

Aguirre, M., Bussolo de Souza, C. and Venema, K., 2016. The gut microbiota from lean and obese subjects contribute differently to the fermentation of arabinogalactan and inulin. PLoS ONE 11: e0159236. https://doi.org/10.1371/journal.pone.0159236

Aguirre, M., Ramiro-Garcia, J., Koenen, M.E. and Venema, K., 2014. To pool or not to pool? Impact of the use of individual and pooled fecal samples for in vitro fermentation studies. Journal of Microbiology Methods 107: 1-7. https://doi.org/10.1016/j.mimet.2014.08.022

Anderson, M.J., 2001. A new method for non-parametric multivariate analysis of variance. Austral Ecology 26: 32-46. https://doi.org/10 .1111/j.1442-9993.2001.01070.pp.x
Blatchford, P., Ansell, J., De Godoy, M.R.C., Fahey, G., Garcia-Mazcorro, J.F., Gibson, G.R., Goh, Y.J., Hotchkiss, A.T., Hutkins, R., LaCroix, C., Rastall, R.A., Reimer, R.A., Schoterman, M., Van Sinderen, D., Venema, K. and Whelan, K., 2013. Prebiotic mechanisms, functions and applications - a review. International Journal of Probiotics and Prebiotics 8: 109-132.

Bottacini, F., Van Sinderen, D. and Ventura, M., 2017. Omics of bifidobacteria: research and insights into their health-promoting activities. Biochemical Journal 474: 4137-4152. https://doi. org/10.1042/BCJ20160756 
Caporaso, J.G., Kuczynski, J., Stombaugh, J., Bittinger, K., Bushman, F.D., Costello, E.K., Fierer, N., Pena, A.G., Goodrich, J.K., Gordon, J.I., Huttley, G.A., Kelley, S.T., Knights, D., Koenig, J.E., Ley, R.E., Lozupone, C.A., McDonald, D., Muegge, B.D., Pirrung, M., Reeder, J., Sevinsky, J.R., Turnbaugh, P.J., Walters, W.A., Widmann, J., Yatsunenko, T., Zaneveld, J. and Knight, R., 2010. QIIME allows analysis of high-throughput community sequencing data. Nature Methods 7: 335-336. https://doi.org/10.1038/nmeth.f.303

Cuevas-Tena, M., Alegria, A., Lagarda, M.J. and Venema, K., 2019. Impact of plant sterols enrichment dose on gut microbiota from lean and obese subjects using TIM-2 in vitro fermentation model. Journal of Functional Foods 54: 164-174. https://doi.org/10.1016/j. jff.2019.01.005

Gibson, G.R., Hutkins, R., Sanders, M.E., Prescott, S.L., Reimer, R.A., Salminen, S.J., Scott, K., Stanton, C., Swanson, K.S., Cani, P.D., Verbeke, K. and Reid, G., 2017. Expert consensus document: the International Scientific Association for Probiotics and Prebiotics (ISAPP) consensus statement on the definition and scope of prebiotics. Nature Reviews Gastroenterology \& Hepatology 14: 491-502. https://doi.org/10.1038/nrgastro.2017.75

Hamer, H.M., Jonkers, D., Venema, K., Vanhoutvin, S., Troost, F.J. and Brummer, R.J., 2008. Review article: the role of butyrate on colonic function. Alimentary Pharmacology \& Therapeutics 27: 104-119. https://doi.org/10.1111/j.1365-2036.2007.03562.x

Hidalgo-Cantabrana, C., Delgado, S., Ruiz, L., Ruas-Madiedo, P., Sanchez, B. and Margolles, A., 2017. Bifidobacteria and their health-promoting effects. Microbiology Spectrum 5(3). https:// doi.org/10.1128/microbiolspec.BAD-0010-2016

Kovatcheva-Datchary, P., Egert, M., Maathuis, A., Rajilic-Stojanovic, M., De Graaf, A.A., Smidt, H., De Vos, W.M. and Venema, K., 2009. Linking phylogenetic identities of bacteria to starch fermentation in an in vitro model of the large intestine by RNA-based stable isotope probing. Environmental Microbiology 11: 914-926. https:// doi.org/10.1111/j.1462-2920.2008.01815.x

Maathuis, A.J., Van den Heuvel, E.G. Schoterman, M.H. and Venema, K., 2012. Galacto-oligosaccharides have prebiotic activity in a dynamic in vitro colon model using a (13)C-labeling technique. Journal of Nutrition 142: 1205-1212. https://doi.org/10.3945/ jn. 111.157420
Minekus, M., Smeets-Peeters, M., Bernalier, A., Marol-Bonnin, S., Havenaar, R., Marteau, P., Alric, M., Fonty, G. and Huis in't Veld, J.H., 1999. A computer-controlled system to simulate conditions of the large intestine with peristaltic mixing, water absorption and absorption of fermentation products. Applied Microbiology and Biotechnology 53: 108-114.

Morrison, D.J., Mackay, W.G., Edwards, C.A., Preston, T., Dodson, B. and Weaver, L.T., 2006. Butyrate production from oligofructose fermentation by the human faecal flora: what is the contribution of extracellular acetate and lactate? British Journal of Nutrition 96: 570-577.

R Core Team, 2013. R: A language and environment for statistical computing. R Foundation for Statistical Computing, Vienna, Austria. Available at: http://www.R-project.org

Slavin, J.L., Brauer, P.M. and Marlett, J.A., 1981. Neutral detergent fiber, hemicellulose and cellulose digestibility in human subjects. Journal of Nutrition 111: 287-297. https://doi.org/10.1093/jn/111.2.287

Van Nuenen, H.M.C., Meyer, P.D. and Venema, K., 2003. The effect of various inulins and Clostridium difficile on the metabolic activity of the human colonic microbiota in vitro. Microbial Ecology in Health and Disease 15: 137-144.

Venema, K. and Van den Abbeele, P., 2013. Experimental models of the gut microbiome. Best Practice \& Research: Clinical Gastroenterology 27: 115-126. https://doi.org/10.1016/j.bpg.2013.03.002

Venema, K., Van Nuenen, H.M.C., Smeets Peeters, M.J.E., Minekus, M. and Havenaar, R., 2000. TNO's in vitro large intestinal model: an excellent screening tool for functional food and pharmaceutical research. Ernährung/Nutrition 24: 558-564.

Venema, K., Van Nuenen, H.M.C., Van den Heuvel, E.G. Pool, W. and Van der Vossen, J.M.B.M., 2003. The effect of lactulose on the composition of the intestinal microbiota and short-chain fatty acid production in human volunteers and a computer-controlled model of the proximal large intestine. Microbial Ecology in Health and Disease 15: 94-105. 